\title{
Adaptation to family-based treatment for Medicaid-insured youth with anorexia nervosa in publicly-funded settings: Protocol for a mixed methods implementation scale-out pilot study
}

Erin C. Accurso ${ }^{1 *} \mathbb{0}$, Karen J. Mu ${ }^{1,2}$, John Landsverk ${ }^{3}$ and Joseph Guydish ${ }^{1,4}$

\begin{abstract}
Background: Family-based treatment (FBT) for anorexia nervosa is an evidence-based treatment, but its effectiveness is untested among socioeconomically disadvantaged and racially diverse youth. Adapting FBT may facilitate "scale-out" for Medicaid-insured youth served in publicly-funded settings and potentially improve outcomes for more diverse populations.
\end{abstract}

Methods: This mixed methods effectiveness-implementation Hybrid Type 3 pilot study protocol included a planning period in collaboration with the San Francisco Department of Public Health, culminating in a two-day in-person FBT training for 25 therapists in the county, followed by the opportunity to engage in one year of weekly supervision. The training incorporated FBT adaptations intended to improve fit for low-income families within community-based settings. Treatment appropriateness and acceptability will be measured immediately post-training. Following the training, cases referred for FBT will only be assigned to the trained clinicians who voluntarily opted into long-term group supervision. Clinicians treating at least one FBT case during the supervision period will report on implementation, adaptations, and patient weight gain. Finally, semi-structured interviews with clinician participants will be conducted, focused on implementation challenges and facilitators, local treatment adaptations, and overall satisfaction with FBT.

Discussion: Learning about clinician adaptations will advance knowledge about treatment of eating disorders in publicly-funded community clinics, which serve a racially/ethnically and socioeconomically diverse group of youth. This project is designed to accelerate FBT implementation in publicly-funded mental health systems, and inform service improvements for underserved youth with eating disorders.

Keywords: Adaptation, Family-based treatment (FBT), Implementation, Mixed methods, Hybrid effectivenessimplementation Type 3 design, "Scale-out", Children and adolescents, Anorexia nervosa, Atypical anorexia nervosa, Publicly-funded settings, Protocol

*Correspondence: erin.accurso@ucsf.edu

${ }^{1}$ Department of Psychiatry and Behavioral Sciences, Weill Institute for Neurosciences, University of California, San Francisco, CA, USA

Full list of author information is available at the end of the article

\section{Background}

Despite a number of evidence-based psychosocial treatments for mental health disorders, implementation is lagging, with a delay of 17 years for treatments to move from research to community-based settings permits use, sharing, adaptation, distribution and reproduction in any medium or format, as long as you give appropriate credit to the original author(s) and the source, provide a link to the Creative Commons licence, and indicate if changes were made. The images or other third party material in this article are included in the article's Creative Commons licence, unless indicated otherwise in a credit line to the material. If material is not included in the article's Creative Commons licence and your intended use is not permitted by statutory regulation or exceeds the permitted use, you will need to obtain permission directly from the copyright holder. To view a copy of this licence, visit http://creativecommons.org/licenses/by/4.0/. The Creative Commons Public Domain Dedication waiver (http://creativeco mmons.org/publicdomain/zero/1.0/) applies to the data made available in this article, unless otherwise stated in a credit line to the data. 
[1] and little regulatory oversight to ensure that evidence-based standards for psychosocial care are met [2]. Implementation science can improve the uptake of effective treatments into routine clinical settings [3]. Economically disadvantaged youth have poor access to evidence-based treatments, and their implementation is more complex because these treatments are often not tested with low-income, racially/ethnically diverse patients served in the public sector [4]. Generally, implementation efforts in mental health treatments have focused on more common concerns (e.g., mood/ anxiety, disruptive behavior), which are likely to have the broadest impact. However, implementation science approaches have been applied infrequently to youth with lower base rate disorders, including those with eating disorders.

Eating disorders have the highest mortality rate of any psychiatric disorder due to high suicide rates [5] and medical instability, including cardiovascular, pulmonary, and gastrointestinal complications, as well as electrolyte abnormalities requiring medical intervention [5-7]. Anorexia nervosa (AN) is particularly precarious. Approximately $20 \%$ of those with AN require medical hospitalization, and $40 \%$ require re-hospitalization within one year [8]. Early, effective treatment is important for AN because of the high mortality rate [9], potential for a chronic course [10], and association between shorter duration of illness and better outcomes [11]. Data indicate that adolescents with atypical AN (AAN), who present at a normal or above normal weight (versus underweight), are just as medically compromised [12]. However, youth with eating disorders do not typically receive evidence-based treatment [13], leaving them more vulnerable to hospitalization.

Family-based treatment (FBT) for adolescents with AN is the most studied treatment for adolescent eating disorders to-date, supported by four randomized trials [14-17]. It is arguably the only adolescent eating disorder treatment meeting the Level 1 "Well-Established Treatment" criteria for dissemination [18]. Implementation of FBT has been confined to specialized eating disorder programs, including settings in national health care systems such as Australia [19] and Canada [20-22], and privately-funded settings in the U.S. [23, 24]. However, there are no published reports focused on "scaling-out" FBT in publicly-funded settings outside of national health care systems, or in non-specialist community-based settings. In the U.S., FBT has been employed primarily with highly educated, White, English-speaking families. Its effectiveness is untested among socioeconomically disadvantaged youth in publicly-funded settings, who are particularly vulnerable in a system that is under-resourced and impacted by health disparities.
While the prevalence of eating disorders in publiclyinsured youth is unknown, prevalence rates are similar across racial/ethnic groups [25], and rates of eating disorder behaviors are similar across socioeconomic levels, but increasing at a faster rate in lower socioeconomic status groups [26]. However, there is a media bias insinuating that eating disorders primarily affect affluent White cisgender females. Gender and sexual minorities are also underrepresented in eating disorders research, despite having heightened rates of eating pathology [27]. As a result, clinicians in publicly-funded settings may be unaware that eating disorders are relevant for the populations with whom they work, which leads to lack of training and investment in diagnosing and treating eating disorders [28]. This may be exacerbated for racial/ethnic minority youth, who have lower rates of mental health service utilization for eating disorders than Whites [29].

Fidelity of an intervention (i.e., the extent to which an intervention is implemented as intended) predicts patient outcomes [30-33], but adaptations-rather than strict adherence-may be required to facilitate implementation and sustainability [34, 35]. FBT is inherently flexible, as it relies on caregivers' expertise about their family to guide recovery. However, some of the core tenets of FBT are difficult to implement in lower-income populations, such as the expectation that caregivers monitor all meals/ snacks.

Meta-analyses show that cultural adaptation improves treatment relevance, acceptability, effectiveness, and sustainability [36, 37]. If not adapted, interventions may meet with therapist resistance and have poor organizational fit $[38,39]$. Clinicians report intention to "drift" from manualized FBT immediately post-training [40], and actual drift has been observed in real-world practice [13, 41]. While FBT principles have been incorporated into higher level of care programs [42-45], little work has been done to adapt FBT, which is an outpatient treatment, to community-based outpatient settings. Adaptations have been made for at least one new population (i.e., young adults) [46, 47], for cultural reasons (Japan) [48], and to improve fit within a community-based setting $[23,49]$. However, adaptations have not been examined for more racially/ethnically and linguistically diverse populations, or for low-income families in publiclyfunded settings.

This article describes the implementation study protocol intended to "scale-out" [50] FBT for use by a new population of therapists (community-based clinicians) with a new population of patients (racially/ethnically diverse, lower-income families) in a new setting (publicly-funded clinics). Cultural adaptations are critical for improving service delivery [51-53] because clinicians are more likely to adopt and implement FBT with high 
fidelity if they perceive the intervention as acceptable for their setting and population [23]. This project will use mixed methods to identify planned and naturally- occurring adaptations to FBT as implemented in publiclyfunded clinics, with the long-term goal to increase health equity and improve publicly-funded services for youth with eating disorders.

\section{Methods/design}

The implementation process in this study was informed by Aarons et al's [54] dynamic adaptation process (DAP) model, which guides the implementation process in a four-phased iterative model Through active engagement with multiple stakeholders, adaptations are made proactively in a way that honors the core components and/or principles of the evidence-based intervention. Elements of the exploration, preparation, and implementation phases, as guided by the Dynamic Adaptation Process, are described below. The study protocol was designed to measure outcomes during the implementation phase (post-training) outlined in the DAP, including implementation outcomes and to a lesser extent patient outcomes. The study design most closely follows Curran et al's [55] conceptualization of a hybrid effectiveness-implementation design, which prioritizes the examination of both clinical effectiveness and implementation outcomes, rather than more exclusively focusing on one or the other. Blending design elements of clinical effectiveness trials and implementation trials is viewed as beneficial and efficient, including Type 1 (i.e., examining clinical effectiveness while observing implementation outcomes), Type 2 (i.e., equal value given to examining clinical effectiveness and testing implementation strategies), and Type 3 hybrids (i.e., testing implementation strategies while observing clinical outcomes) [55]. Given this study's focus on implementation strategies while observing and gathering some more limited data on patient outcomes, this protocol best reflects a Hybrid Type 3 design. Standards for Reporting Implementation Studies (StaRI) were used to guide this report $[56,57]$.

\section{Context of the implementation setting}

In December 2018, the San Francisco Department of Public Health (DPH) reached out to the first author to work on coordinating a date for FBT training given increasing numbers of youth with AN and AAN presenting for treatment in the county. Prior to the training, DPH administrators identified five publicly-funded mental health agencies that provided intensive services. Each of the agencies was informed about the two-day training and year-long supervision program via email. Three of these programs were targeted for additional outreach due to their strategic geographic location across the county and Spanish language capacity, given county-level data indicating that $60 \%$ of eating disorder cases in the year prior to training were Latinx, most of whom were Spanish-speaking. Meetings between two of the authors (ECA, KJM) and directors of the three targeted agencies were held to discuss the logistics of the two-day training and the one-year supervision program.

Directors then discussed the training with clinicians, and one author (KJM) presented at each clinic about the goals of the training and supervision program. A brief clinician survey completed at these meetings indicated minimal exposure to eating disorders and enthusiasm for serving a new population. Clinicians revealed reservations about whether FBT could be applied to a Medicaid-insured population, given that it had been largely tested in university-based medical centers or specialized community-based settings. An invitation to the training was extended by DPH administrators to a sixth agency the day prior to the training in order to balance the number of "internal" agencies and community-based agencies attending.. All study procedures were approved by the IRB at the University of California, San Francisco.

\section{Two-day FBT training}

In total, 25 clinicians from six agencies in San Francisco County attended the two-day in-person FBT training in September 2019, including clinicians from the three targeted agencies. The first and second authors (ECA and KJM) led the training, in partnership with local expert FBT clinicians providing publicly-funded services, followed by the opportunity for clinicians to engage in one year of weekly FBT supervision. Languages spoken by clinicians who attended the training included Spanish, Cantonese, Mandarin, and Russian. The first half-day provided a background on eating disorders, including assessment across the spectrum of eating disorders. The remainder of the first day and second day were devoted specifically to FBT for AN and AAN consistent with the FBT manual [58], incorporating didactic presentations, role plays, and discussion [58]. It also incorporated several adaptations designed to improve fit for low-income families. Table 1 provides an overview of content adaptations that were planned during the pre-implementation phase [59]. Ultimately, the first author led decisions on modifications based on her expertise in FBT and knowledge of the clinical population, including feedback from clinicians during the training-to the extent that modifications preserved core elements and functions of the intervention. In addition to modifications of training content, a more global change was made to the training itself, in order to improve clinician-reported intervention acceptability and appropriateness and increase clinician satisfaction with training. The first half-day of 


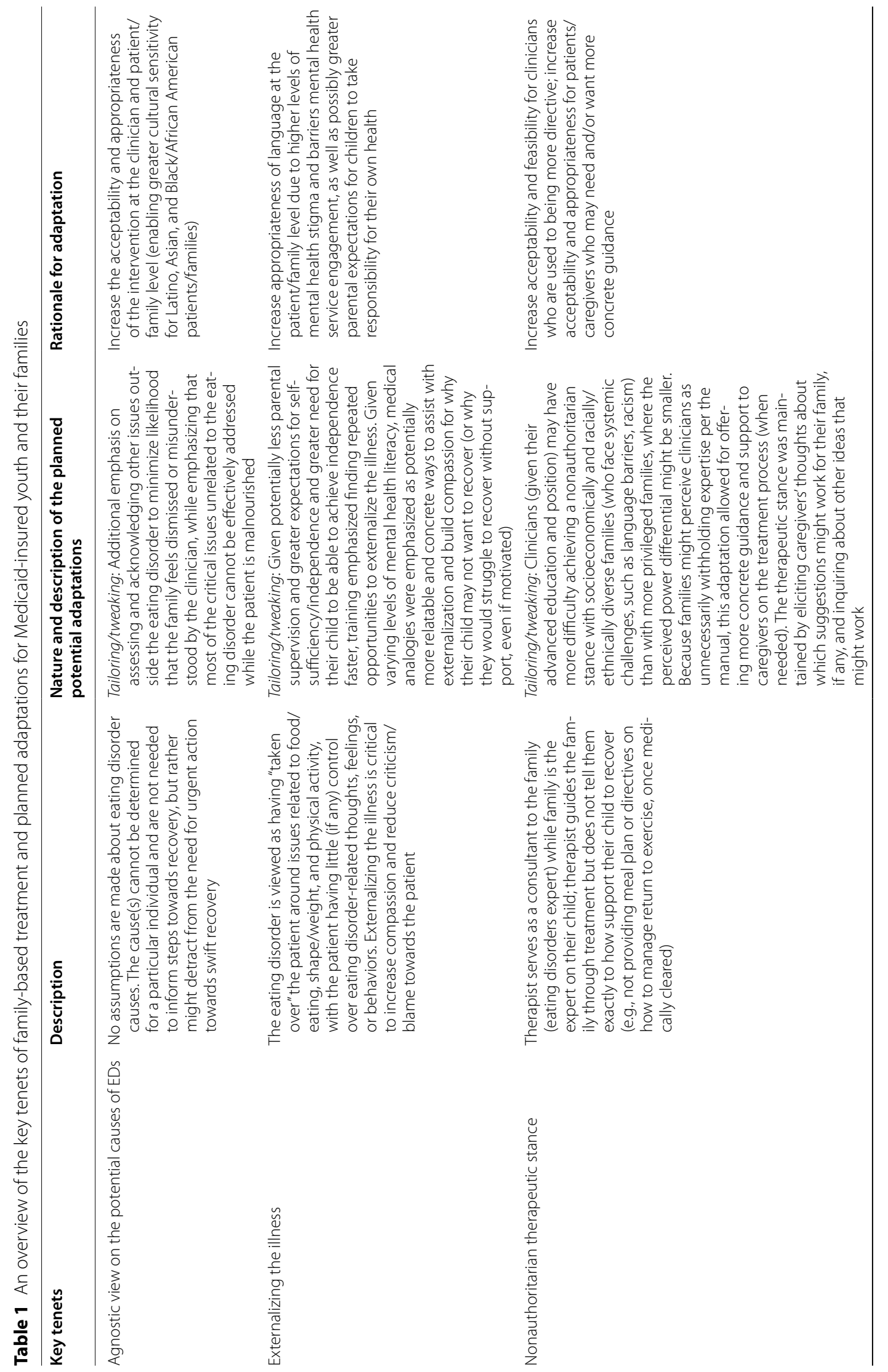




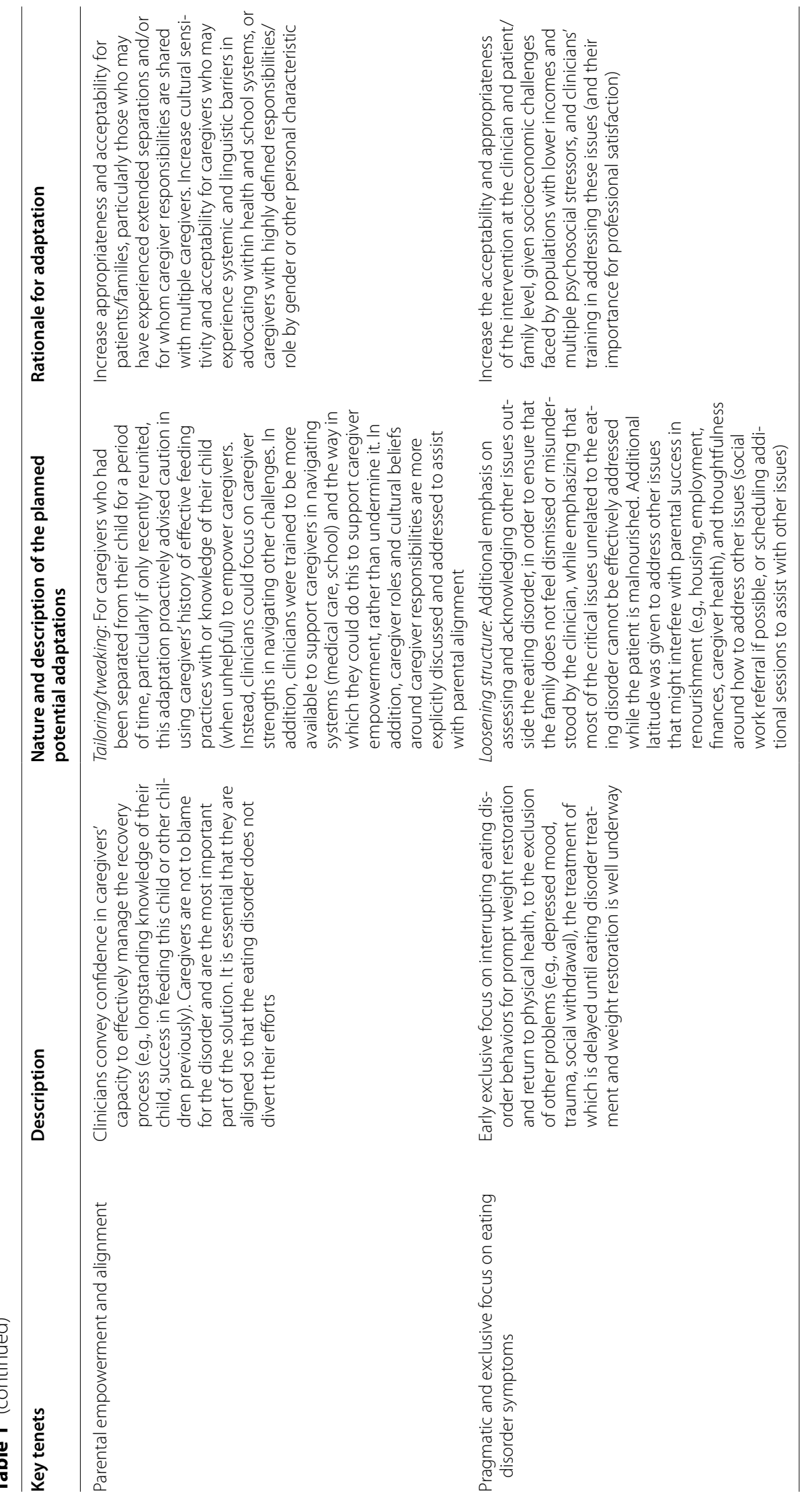


standard FBT training would typically review FBT efficacy research findings. Instead, our training provided approximately three hours of training in the assessment of eating disorders (given that our clinician population had limited experience in eating disorders), with brief research presentations on eating disorders in publiclyinsured populations and the evidence base for FBT. Further, time was reserved at the end of the training to address clinician concerns about implementation and to collaboratively discuss further adaptations. This is in contrast to standard FBT training, where trainers respond to questions about adaptations by reassuring trainees that novice FBT clinicians often have initial concerns about the feasibility and acceptability of particular intervention components but actively discouraging any departures from the manualized intervention.

\section{Planned potential adaptations}

Parental empowerment is a central tenet of FBT. Caregivers are charged with managing eating disorder behavior in the first phase of treatment, including all decisions around nourishment (e.g., what foods, when, how much) and personal responsibility for supervising all meals and snacks. These activities demand a level of parental involvement that is often not feasible for single caregivers, or caregivers with demanding work schedules, inflexible work hours, or limited financial resources. As an adaptation, clinicians were trained to empower caregivers to be resourceful in making a meal/snack supervision plan while being more flexible in brainstorming who could provide supervision. Although the manual is explicit that siblings should not assist with supervising meals, a sibling's role in supporting the patient warrants a more nuanced discussion when families are already depending on older siblings in a parenting capacity. Brainstorming might also include participation from extended family members, family friends, or neighbors as part of the system supporting renourishment efforts.

FBT discussions would also include problem-solving around how to manage snacks and lunch during school hours. Ideally, one caregiver would be available to meet their child for lunch at school, or alternatively caregivers would identify a staff member (nurse, counselor, teacher) who could supervise meals/snacks at school. Rather than empowering caregivers to manage these conversations independently, training allowed for clinicians to empower caregivers by more directly facilitating or supporting caregivers in navigating these conversations and included greater emphasis how to collaborate with school personnel to aid in monitoring lunch/snacks (e.g., requesting accommodations for meal/snack supervision within school setting).
In FBT, there is an expectation that caregivers may contribute in different ways to renourishment efforts, but that parental alignment around their approach is critical, and the importance of balanced contributions so that one caregiver does not carry full responsibility. The adapted training discussed cultural differences in parenting and gender roles in the family, with explicit discussion of caregiver roles in the home and around parenting, and thinking about how to work within an existing system or discussing a reorganized family system needed to support their child. The adapted training emphasized the balance between active engagement from all caregivers and realistic expectations about what level of engagement might be possible. In manualized FBT, sessions are typically rescheduled if any caregiver is absent from a session. Doing so with Medicaid-insured patients and their families risks damaging rapport and signals a lack of respect for the efforts and expense taken by those who did arrive for the appointment. Clinicians were instructed to work with the family that show up for the appointment, but to continue efforts to engage all caregivers, even if engagement meant a brief update by phone during or after sessions.

FBT asks families to "externalize the illness," an abstract concept meant to help families understand that initially patients have almost no control over their eating disorder. In the adapted training clinicians were trained to invest more time on this concept, as the capacity to externalize the illness is critical to caregiver success in renourishment and in their ability to empathize with their child while remaining firm with the eating disorder. Additional time was spent discussing possible analogies (e.g., eating disorder as a tumor) that might help families understand this concept. These adaptations were intended to increase implementation flexibility without changing core elements related to treatment effectiveness [60].

Finally, clinician reservations about the treatment approach and their concerns about its applicability to their treatment population were directly addressed. Trainers acknowledged that FBT has not been studied in Medicaid-insured populations while emphasizing that FBT has been shown to work more quickly and effectively than alternative treatments $[14,16,61]$. The two local FBT clinicians shared clinical case examples and conveyed confidence about FBT as a treatment approach for Medicaid-insured youth with AN and AAN. Trainers also invited participants to leverage their expertise with this population to adapt FBT for publicly-funded settings. One concern discussed was families' capacity to engage in weekly office-based therapy, given that agencies provided a mix of office- and home-based services and had extensive experience engaging with families "in the field." The trainers discussed home-based visits as an 
adaptation, but several clinicians from different agencies raised concerns that home-based visits might undermine the importance of treatment. Rather, they believed that having families come into the office (rather than clinicians going to the family) would assist families in prioritizing treatment, lend structure and containment to visits that would have been difficult outside of the office, and help manage safety concerns (e.g., access to a provider who could take patient vitals, if indicated). Clinicians discussed how they decided only to deliver dialectical behavior therapy [62] in the office, for similar clinical and safety reasons. Clinical supervisors present at the meeting reached consensus to generally abstain from engaging in home-based visits given these concerns.

\section{Participants and procedures}

Immediately post-training, clinicians will report on appropriateness and acceptability of FBT as an approach for Medicaid-insured youth with AN or AAN. These are key when considering cultural adaptations [63]. Clinician recruitment, with a goal of recruiting 10 clinicians, is in progress. Given the pilot nature of this study and county size, target enrollment is 10 clinicians in order to achieve data saturation for qualitative research. Each clinician who opts into group supervision will receive a copy of the FBT manual [58] and be invited to participate in the study. The recruitment target was also influenced by practical considerations, including resources required to prepare a behavioral health county to identify and refer eating disorder cases. Supervision will occur weekly for approximately one year post-training. Naturally occurring ad hoc adaptations (i.e., those not planned in the initial training) will be discussed on an ongoing basis during supervision. For FBT sessions conducted during the one-year supervision period, clinicians will report on implementation of FBT techniques, note adaptations, and measure patient weight.

\section{Post-training assessment}

Immediately following the two-day training, clinicians will complete quantitative measures.

FBT Knowledge Assessment (FBT-KA). The FBT-KA is an 18-item multiple-choice measure co-developed by the first author that assesses knowledge about FBT for AN and AAN and reviewed/approved by one of the treatment developers.

FBT Attitude Scale (FBT-AS) [40]. The FBT-AS is a 20-item measure of clinician attitudes towards FBT. The FBT-AS has good reliability and validity, including predicting intent to change practice to be more consistent with FBT [40].

Treatment Evaluation Inventory Short Form (TEI-SF) [64]. The TEI-SF is a 9-item clinician-reported measure of treatment acceptability with good reliability and validity. Instructions requested that clinicians rate each item with respect to how they felt about using FBT for eating disorder behaviors in the context of anorexia nervosa or atypical anorexia nervosa. Generic language in the items was modified (e.g., this treatment, problem behavior, and child were replaced with $F B T$, eating disorder, and client.

\section{Active treatment and post-supervision assessment}

Clinicians in the one-year supervision cohort who have an active FBT case will complete additional measures. Regardless of whether they have an opportunity to treat an FBT case, they will be invited to participate in a qualitative semi-structured interview as detailed below. Other outcomes, including an economic evaluation, were outside the scope of this pilot study.

De-identified patient data. Clinicians will provide deidentified patient/caregiver demographics, diagnosis, session dates, and weights/heights taken by the clinician in the context of treatment.

FBT Therapeutic Techniques Scale (FBT-TTS). [23] The FBT-TTS is a clinician self-report measure of FBT technique implementation completed after each FBT session through six months. Alongside their reported use of FBT techniques, clinicians will be asked to record in real-time any adaptations they make to FBT.

Qualitative semi-structured interviews. Approximately one year after FBT is initiated with the first referred case, the first author will conduct semi-structured interviews with clinician participants to learn about implementation challenges and facilitators, naturally occurring treatment adaptations, including rationale and perceived impact on outcome, and overall satisfaction with FBT. Openended interview questions will be guided by the five-part, multi-level Consolidated Framework for Implementation Research (CFIR) [65] and the Capability, Opportunity, Motivation, Behaviour (COM-B) model [66]. CFIR considers characteristics of the intervention, outer setting, inner setting, characteristics of the individuals involved, and the implementation process, whereas COM-B focuses on how capability (physical and psychological), opportunity (physical and social), and motivation influence behavior change. The interview guide will be piloted and revised prior to conducting the first interview and will continue to be refined as needed throughout data collection.

\section{Statistical analysis}

The post-training measures will be analyzed using SPSS to describe FBT-related knowledge (FBT-KA), attitudes (FBT-AS), and treatment acceptability (TEI-SF). Measures completed during treatment, will also be analyzed and summarized using SPSS, including self-reported 
implementation of FBT techniques (FBT-TTS) and patient data. Exploratory, descriptive statistics will be used to examine quantitative data on attitudes and acceptability, given the cohort design with a small sample, which is not sufficiently powered to examine change over time.

Semi-structured qualitative interviews will be transcribed and uploaded into qualitative analysis software (Dedoose [67, 68], exploring themes about treatment acceptability, barriers and facilitators to implementation, and resulting adaptations. Stirman et al's [59] Framework for Reporting Adaptations and Modifications-Expanded (FRAME) will be used for categorizing changes into contextual modifications (e.g., changes to format or setting) or content modifications (i.e., changes to "how" or "what" intervention content is delivered). These modifications will be evaluated on the extent to which they represent fidelity-consistent and fidelity-inconsistent adaptations [69]. Trained staff will independently review and code interview transcripts to identify and abstract themes following standardized procedures. Each interview will be reviewed by two researchers, from which a list of emerging themes and codes will be developed. These codes will then be independently applied to each interview by both researchers. Coding meetings will be held during the coding process to identify and codify new themes and resolve coding discrepancies. Qualitative findings on implementation and adaptations will be integrated with quantitative data on the implementation, treatment acceptability, and attitudes towards FBT. Preliminary outcome data will be presented.

\section{Discussion}

This hybrid protocol is designed to "scale out" standard FBT (training and implementation)-adapting training and the intervention itself for clinicians in publiclyfunded community settings working with more diverse service populations, with the ultimate goal of increasing access to acceptable and appropriate evidence-based eating disorder treatment for underserved youth. Given that eating disorders are increasingly recognized in a range of service populations [26], adapting evidence-based eating disorder treatments for use in community-based clinics is essential. Data collection is ongoing, currently focused on completing semi-structured interviews with clinician participants to learn about implementation challenges, acceptability of FBT, and local treatment adaptations. Studying how evidence-based treatments are adapted to improve their fit in real world settings or for diverse service populations is important to advancing the science of adaptation in implementation [70]. These adaptations will inform a web-based training in "real world" FBT, which is currently under development. Online training has significant advantages over traditional in-person training as an accessible, flexible, scalable, and sustainable method of training [71, 72]. Online training has been successfully disseminated for evidence-based eating disorder treatments previously [73, 74] and its advantages over standard face-to-face training extend to underresourced systems with high clinician turnover treating low base-rate disorders such as eating disorders.

\section{Limitations}

This is one of the earliest efforts to study implementation of a treatment for Medicaid-insured youth with eating disorders, but there are several limitations. First, this study includes a limited sample of clinicians treating patients within one county due to practical constraints of preparing a system of care to manage eating disorder patients in coordinated interdisciplinary care. Second, due to the changing context of care with the onset of the Covid-19 pandemic, this team decided that it would not be feasible to engage families in an observational research study when it was evident from supervision that families were struggling to engage in mental health treatment due to pandemic-related challenges. As a result, we modified the protocol to collect de-identified patient data, rather than prospectively collecting more comprehensive patient and family outcomes. Third, and also related to the pandemic, we did not collect session recordings due to the challenges of recording clinical sessions in a virtual environment, including the fact that clinicians were using personal, non-encrypted devices to conduct sessions. As a result, we collected self-report data on implementation, including adaptations, without being able to directly observe and code clinician behavior. Finally, this study is limited by its traditional resource-intensive approach to training (i.e., in-person training conducted over multiple days with follow-up supervision), which cannot be reasonably scaled up.

\section{Impact}

This project will develop a pathway to accelerate implementation efforts in under-resourced publicly-funded mental health systems and inform service improvements for high-risk youth with AN and AAN seeking treatment in community-based settings. Learning about clinician adaptations will advance knowledge about how to treat eating disorders in publicly-funded community clinics, which serve youth who are more racially/ethnically diverse youth than those with whom the interventions have been tested to date. Given the potential benefits of telehealth over traditional in-person services for specialty mental health, lessons on how clinicians additionally adapted FBT in order to adjust to providing treatment via telehealth to lower-income families in the context 
of the pandemic may be particularly informative. Additionally, we will explore stakeholder attitudes towards FBT and facilitators and barriers to implementation in these settings. Building on the learning from this study, we aim to refine the adapted treatment model to better fit Medicaid-insured youth with eating disorders and settings in which they are served. Studying implementation challenges in the "real world" may also help to identify further refinements to FBT that could improve outcomes more broadly for youth eating disorders across sociodemographic levels and settings. Further, this study may provide context for some of the contextual challenges of implementing interventions in publicly-funded settings (e.g., administrative time needed to access resources, including treatment manuals). A byproduct of this implementation effort may be greater investment in improved screening and treatment of eating disorders, particularly if administrative staff and clinicians trust that these patients-once identified-can be successfully treated with a model that fits well with this population and these settings. Ultimately, this line of research aims to improve outcomes, promote health equity, and support future implementation efforts for Medicaid-insured youth with eating disorders.

\begin{abstract}
Abbreviations
AN: Anorexia nervosa; AAN: Atypical anorexia nervosa; FBT: Family-based treatment; DAP: Dynamic adaptation process; StaRI: Standards for reporting implementation studies; DPH: Department of Public Health; CFIR: Consolidated framework for implementation research; COM-B: Capability, opportunity, motivation, behavior; FRAME: Framework for reporting adaptations and modifications-expanded.
\end{abstract}

\section{Acknowledgements}

We would like to acknowledge the tremendously skilled clinicians who were generous with their time and expertise as study participants, as well as the San Francisco Department of Public Health for supporting this training and research effort.

\section{Authors' contributions}

All authors listed have made a substantial, direct and intellectual contribution to the work, and approved it for publication. ECA conceptualized the study with JG and JL. KJM assisted with the preparation and implementation phases of the study protocol. All authors read and approved the final manuscript.

\section{Funding}

This study is funded by the National Institute of Mental Health (NIMH K23

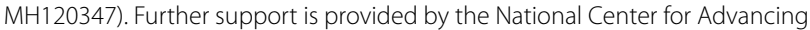
Translational Sciences, National Institutes of Health, through UCSF-CTSI Grant Number KL2 TR001870. Its contents are solely the responsibility of the authors and do not necessarily represent the official views of the $\mathrm{NIH}$.

\section{Availability of data and materials}

Not applicable.

\section{Declarations}

\section{Ethical approval and consent to participate}

The studies involving human participants were reviewed and approved by the Institutional Review Board at the University of California, San Francisco. Participants will provide written informed consent to participate in this study.

\section{Consent for publication}

Not applicable.

\section{Competing interests}

The first author consults with Partnership HealthPlan of California (a health care organization that contracts with the State to administer Medicaid benefits) concerning strategies to improve the treatment of eating disorders. The authors deny any other commercial or financial relationships that could be construed as a potential conflict of interest.

\section{Author details}

${ }^{1}$ Department of Psychiatry and Behavioral Sciences, Weill Institute for Neurosciences, University of California, San Francisco, CA, USA. ${ }^{2}$ San Francisco Department of Public, Health Behavioral Health Services, San Francisco, CA, USA. ${ }^{3}$ Oregon Social Learning Center, Eugene, OR, USA. ${ }^{4}$ Philip R. Lee Institute for Health Policy Studies, University of California, San Francisco, CA, USA.

Received: 19 May 2021 Accepted: 4 Auqust 2021

Published online: 13 August 2021

\section{References}

1. Morris ZS, Wooding S, Grant J. The answer is 17 years, what is the question: Understanding time lags in translational research. J R Soc Med. 2011;104(12):510-20.

2. Committee on Developing Evidence-Based Standards for Psychosocial Interventions for Mental Disorders; Board on Health Sciences Policy; Institute of Medicine; England MJ, Butler AS, Gonzalez ML, editors. Psychosocial interventions for mental and substance use disorders: A framework for establishing evidence-based standards. Washington (DC): National Academies Press (US); 2015. https://doi.org/10.17226/19013.

3. Bauer MS, Kirchner JA. Implementation science: What is it and why should I care? Psychiatry Res 2020;283:112376.

4. Stadnick NA, Aarons GA, Blake L, Brookman-Frazee LI, Dourgnon P, Engell $T$, et al. Leveraging implementation science to reduce inequities in children's mental health care: Highlights from a multidisciplinary international colloquium. BMC Proc. 2020;14(S2):2.

5. Crow SJ, Peterson CB, Swanson SA, Raymond NC, Specker S, Eckert ED, et al. Increased mortality in bulimia nervosa and other eating disorders. Am J Psychiatry. 2009;166(12):1342-6.

6. Mitchell JE, Crow S. Medical complications of anorexia nervosa and bulimia nervosa. Curr Opin Psychiatry. 2006;19:438-43.

7. Andersen AE, Yager J. Eating disorders. In: Sadock BJ, Sadock VA, Ruiz P, editors. Kaplan \& Sadock's comprehensive textbook of psychiatry, vol. I. 9th ed. Philadelphia: Lippincott Williams \& Wilkins; 2009. p. 2128-49.

8. Steinhausen HC, Grigoroiu-Serbanescu M, Boyadjieva S, Neumärker KJ, Metzke CW. Course and predictors of rehospitalization in adolescent anorexia nervosa in a multisite study. Int J Eat Disord. 2008;41 (1):29-36.

9. Arcelus J, Mitchell AJ, Wales J, Nielsen S. Mortality rates in patients with anorexia nervosa and other eating disorders. Arch Gen Psychiatry. 2011;68(7):724-31.

10. Steinhausen HC. The outcome of anorexia nervosa in the 20th century. Am J Psychiatry. 2002;159(8):1284-93.

11. Le Grange D, Lock J, Agras WS, Moye A, Bryson SW, Jo B, et al. Moderators and mediators of remission in family-based treatment and adolescent focused therapy for anorexia nervosa. Behav Res Ther. 2012;50(2):85-92.

12. Golden NH, Mehler PS. Atypical anorexia nervosa can be just as bad. Clevel Clin J Med. 2020;87:172-4.

13. Couturier J, Kimber M, Jack S, Niccols A, Van Blyderveen S, McVey G. Understanding the uptake of family-based treatment for adolescents with anorexia nervosa: therapist perspectives. Int J Eat Disord. 2013;46(2):177-88.

14. Agras WS, Lock J, Brandt H, Bryson SW, Dodge E, Halmi KA, et al. Comparison of two family therapies for adolescent anorexia nervosa: a randomized parallel trial. JAMA Psychiat. 2014;71(11):1279-86.

15. le Grange D, Hughes EK, Court A, Yeo M, Crosby RD, Sawyer SM. Randomized clinical trial of parent-focused treatment and family-based treatment for adolescent anorexia nervosa. J Am Acad Child Adoles Psychiatry. 2016;55(8):683-92. 
16. Lock J, Le Grange D, Agras WS, Moye A, Bryson SW, Jo B. Randomized clinical trial comparing family-based treatment with adolescent-focused individual therapy for adolescents with anorexia nervosa. Arch Gen Psychiatry 2010;67(10):1025-32.

17. Lock J, Agras WS, Bryson S, Kraemer HC. A comparison of short- and longterm family therapy for adolescent anorexia nervosa. J Am Acad Child Adolesc Psychiatry. 2005;44(7):632-9.

18. Lock J. An update on evidence-based psychosocial treatments for eating disorders in children and adolescents. J Clin Child Adoles Psychology. 2015;44(5):707-21.

19. Hughes EK, Le Grange D, Court A, Yeo M, Campbell S, Whitelaw M, et al. Implementation of family-based treatment for adolescents with anorexia nervosa. J Pediatr Health Care. 2014;28(4):322-30.

20. Couturier J, Kimber M, Lock J, Barwick M, Mcvey G, Findlay S, et al. Implementing highly specialized and evidence-based pediatric eating disorder treatment: protocol for a mixed methods evaluation. Implement Sci. 2015;10:1-7.

21. Couturier J, Kimber M, Barwick M, Woodford T, Mcvey G, Findlay S, et al. Themes arising during implementation consultation with teams applying family-based treatment: a qualitative study. J Eat Disord. 2018;6(1):32.

22. Dimitropoulos G, Landers AL, Freeman V, Novick J, Ma BA, Garber A, et al. Open trial of family-based treatment of anorexia nervosa for transition age youth. J Can Acad Child Adolesc Psychiatry. 2018;27:50.

23. Accurso E, Astrachan-Fletcher E, O'Brien S, McClanahan S, Le Grange D. Adaptation and implementation of family-based treatment enhanced with dialectical behavior therapy skills for anorexia nervosa in community-based specialist clinics. Eat Disord. 2017:26:1-15.

24. Goldstein M, Murray SB, Griffiths S, Rayner K, Podkowka J, Bateman JE, et al. The effectiveness of family-based treatment for full and partial adolescent anorexia nervosa in an independent private practice setting: clinical outcomes. Int J Eat Disord. 2016:49(11):1023-6.

25. Marques L, Alegria M, Becker AE, Chen CN, Fang A, Chosak A, et al. Comparative prevalence, correlates of impairment, and service utilization for eating disorders across US ethnic groups: Implications for reducing ethnic disparities in health care access for eating disorders. Int J Eat Disord. 2011;44(5):412-20.

26. Mitchison D, Hay P, Slewa-Younan S, Mond J. The changing demographic profile of eating disorder behaviors in the community. BMC Public Health. 2014;14(1):943.

27. Simone M, Askew A, Lust K, Eisenberg ME, Pisetsky EM. Disparities in self-reported eating disorders and academic impairment in sexual and gender minority college students relative to their heterosexual and cisgender peers. Int J Eat Disord. 2020;53:513-24.

28. Accurso EC, Buckelew SM, Snowden LR. Youth insured by Medicaid with restrictive eating disorders-underrecognized and underresourced. JAMA Pediatr. 2021; https://doi.org/10.1001/jamapediatrics.2021.2081.

29. Becker AE, Franko DL, Speck A, Herzog DB. Ethnicity and differential access to care for eating disorder symptoms. Int J Eat Disord. 2003;33(2):205-12.

30. Guydish J, Campbell BK, Manuel JK, Delucchi KL, Le T, Peavy KM, et al. Does treatment fidelity predict client outcomes in 12-Step Facilitation for stimulant abuse? Drug Alcohol Depend. 2014;134:330-6.

31. Hukkelberg SS, Ogden T. Working alliance and treatment fidelity as predictors of externalizing problem behaviors in parent management training. J Consult Clin Psychol. 2013;81(6):1010-20.

32. Schoenwald SK, Chapman JE, Sheidow AJ, Carter RE. Long-term youth criminal outcomes in MST Transport: the impact of therapist adherence and organizational climate and structure. J Clin Child Adolesc Psychol. 2009;38(1):91-105.

33. Smith JD, Dishion TJ, Shaw DS, Wilson MN. Indirect effects of fidelity to the Family Check-Up on changes in parenting and early childhood problem behaviors. J Consult Clin Psychol. 2013;81(6):962-74.

34. Barber JP, Gallop R, Crits-Christoph P, Frank A, Thase ME, Weiss RD, et al. The role of therapist adherence, therapist competence, and alliance in predicting outcome of individual drug counseling: results from the National Institute Drug Abuse Collaborative Cocaine Treatment Study. Psychother Res. 2006;16(2):229-40.

35. Hogue A, Dauber S, Chinchilla P, et al. Assessing fidelity in individual and family therapy for adolescent substance abuse. J Subst Abuse Treatm. 2008:35:137-47.
36. Benish SG, Quintana S, Wampold BE. Culturally adapted psychotherapy and the legitimacy of myth: a direct-comparison meta-analysis. J Couns Psychol. 2011;58(3):279-89.

37. Griner D, Smith TB. Culturally adapted mental health intervention: a meta-analytic review. Psychother Theory Res Pract Train. 2006:43(4):531-48.

38. Greenhalgh T, Robert G, Macfarlane F, Bate P, Kyriakidou O. Diffusion of innovations in service organizations: systematic review and recommendations. Milbank Q. 2004;82:581-629.

39. Fixsen D, Naoom S, Blase K, Friedman R, Wallace F. Implementation research: A synthesis of the literature. Tampa, FL: University of South Florida, Louis de la Parte Florida Mental Health Institute, National Implementation Research Network; 2005.

40. Accurso EC, Le Grange D, Graham AK. Attitudes toward family-based treatment impact therapists' intent to change their therapeutic practice for adolescent anorexia nervosa. Front Psych. 2020;23(11):305.

41. Kosmerly S, Waller G, Robinson AL. Clinician adherence to guidelines in the delivery of family-based therapy for eating disorders. Int J Eat Disord. 2015;48(2):223-9.

42. Hoste RR. Incorporating family-based therapy principles into a partial hospitalization programme for adolescents with anorexia nervosa: challenges and considerations. J Fam Ther. 2015;37(1):41-60.

43. Rienecke RD, Wallis E. Ensuring continuity of family-based care across levels of treatment. In: Goldschmidt AB, Le Grange D, editors. Adapting evidence-based eating disorder treatments for novel populations and settings. 1st ed. Routledge; 2020. p. 352-66.

44. Huryk KM, Casasnovas AF, Feehan M, Paseka K, Gazzola P, Loeb KL. Lower rates of readmission following integration of family-based treatment in a higher level of care. Eat Disord. 2020;31:1-8.

45. Martin-Wagar CA, Holmes S, Bhatnagar KAC. Predictors of weight restoration in a day-treatment program that supports familybased treatment for adolescents with anorexia nervosa. Eat Disord. 2019;27(4):400-17.

46. Dimitropoulos G, Freeman VE, Allemang B, Couturier J, Mcvey G, Lock $J$, et al. Family-based treatment with transition age youth with anorexia nervosa: A qualitative summary of application in clinical practice. J Eat Disord. 2015;3(1):1.

47. Chen EY, Weissman JA, Zeffiro TA, Yiu A, Eneva KT, Arlt JM, et al. Familybased therapy for young adults with Anorexia Nervosa restores weight. Int J Eat Disord. 2016:49(7):701-7.

48. Iguchi T, Miyawaki D, Harada T, Ogiwara K. Introduction of family-based treatment to Japan with adaptations to optimize the cultural acceptability and advance current traditional treatments of adolescent anorexia nervosa. Int J Eat Disord. 2021:54(1):117-9.

49. Astrachan-Fletcher E, Accurso EC, Rossman S, McClanahan SF, Dimitropoulos G, Le Grange D. An exploratory study of challenges and successes in implementing adapted family-based treatment in a community setting. J Eat Disord. 2018;6(1):1-9.

50. Aarons GA, Sklar M, Mustanski B, Benbow N, Brown CH. "Scaling-out" evidence-based interventions to new populations or new health care delivery systems. Implement Sci. 2017;12(1):1-13. https://doi.org/10. 1186/s13012-017-0640-6.

51. Baumann AA, Powell BJ, Kohl PL, Tabak RG, Penalba V, Proctor EK, et al. Cultural adaptation and implementation of evidence-based parent-training : a systematic review and critique of guiding evidence. Child Youth Serv Rev. 2015;53:113-20.

52. Bernal G, Jiménez-Chafey MI, Domenech Rodríguez MM. Cultural adaptation of treatments: A resource for considering culture in evidence-based practice. Prof Psychology: Res Pract. 2009;40(4):361-8.

53. Domenech Rodríguez MM, Baumann AA, Schwartz AL. Cultural adaptation of an evidence based intervention: From theory to practice in a Latino/a community context. Am J Community Psychol. 2011;47:170-86.

54. Aarons GA, Green AE, Palinkas LA, Self-Brown S, Whitaker DJ, Lutzker JR, et al. Dynamic adaptation process to implement an evidence-based child maltreatment intervention. Implement Sci. 2012;7(1):32.

55. Curran GM, Bauer M, Mittman B, Pyne JM, Stetler C. Effectiveness-implementation hybrid designs: combining elements of clinical effectiveness and implementation research to enhance public health impact. Med Care. 2012;50(3):217-26. 
56. Pinnock H, Barwick M, Carpenter CR, Eldridge S, Grandes G, Griffiths CJ, et al. Standards for Reporting Implementation Studies (StaRI) Statement. BMJ. 2017;356:16795.

57. Pinnock H, Barwick M, Carpenter CR, Eldridge S, Grandes G, Griffiths CJ, et al. Standards for reporting implementation studies (StaRI): explanation and elaboration document. Open. 2017;7:13318.

58. Lock J, Le Grange D. Treatment manual for anorexia nervosa: a familybased approach. 2nd ed. New York: The Guilford Press; 2015.

59. Stirman SW, Baumann AA, Miller CJ. The FRAME: An expanded framework for reporting adaptations and modifications to evidence-based interventions. Implement Sci. 2019;14(1):1-10.

60. Carvalho ML, Honeycutt S, Escoffery C, Glanz K, Sabbs D, Kegler MC. Balancing fidelity and adaptation: implementing evidence-based chronic disease prevention programs. J Public Health Manag Pract. 2013;19(4):348-56.

61. Le Grange D, Eckhardt S, Dalle Grave R, Crosby RD, Peterson CB, Keery H, et al. Enhanced cognitive-behavior therapy and family-based treatment for adolescents with an eating disorder: A non-randomized effectiveness trial. Psychol Med. 2020;3:1-11.

62. Linehan MM. DBT Skills Training Manual. 2nd ed. New York, NY: The Guilford Press; 2015.

63. Cabassa LJ, Baumann AA. A two-way street: bridging implementation science and cultural adaptations of mental health treatments. Implementation Sci. 2013;8:90.

64. Kelley ML, Heffer RW, Gresham FM, Elliott SN. Development of a modified treatment evaluation inventory. J Psychopathol Behav Assess. 1989;11(3):235-47.

65. Damschroder LJ, Aron DC, Keith RE, Kirsh SR, Alexander JA, Lowery JC. Fostering implementation of health services research findings into prac tice: a consolidated framework for advancing implementation science. Implement Sci. 2009;4(50):40-55.
66. Michie S, Atkins L, West R. The Behaviour change wheel: a guide to designing interventions. London: Silverback Publishing; 2014.

67. Coffey A, Atkinson P. Making sense of qualitative data: complementary research strategies. Thousand Oaks: Sage Publications, Inc; 1996.

68. Denzin NK, Lincoln YS, editors. The SAGE handbook of qualitative research, 3rd edition. Sage Publications Ltd; 2005

69. Wiltsey Stirman S, Gutner CA, Crits-Christoph P, Edmunds J, Evans AC, Beidas RS. Relationships between clinician-level attributes and fidelityconsistent and fidelity-inconsistent modifications to an evidence-based psychotherapy. Implement Sci. 2015;10(1):1-10.

70. Chambers DA, Norton WE. The adaptome: advancing the science of intervention adaptation. Am J Prev Med. 2016;51(4):S124-31.

71. Khanna MS, Kendall PC. Bringing technology to training: web-based therapist training to promote the development of competent cognitivebehavioral therapists. Cogn Behav Pract. 2015;22(3):291-301.

72. Beidas RS, Koerner K, Weingardt KR, Kendall PC. Training research: Practical recommendations for maximum impact. Adm Policy Mental Health Mental Health Serv Res. 2011:38(4):223-37.

73. Fairburn CG, Allen E, Bailey-Straebler S, O'Connor ME, Cooper Z. Scaling up psychological treatments: A countrywide test of the online training of therapists. J Med Internet Res. 2017;19(6):7864.

74. Lock J, Le Grange D, Accurso EC, Welch H, Mondal S, Agras WS. Is online training in family-based treatment for anorexia nervosa feasible and can it improve fidelity to key components affecting outcome? J Behav Cogn Ther. 2020;30(2):75-82.

\section{Publisher's Note}

Springer Nature remains neutral with regard to jurisdictional claims in published maps and institutional affiliations.
Ready to submit your research? Choose BMC and benefit from:

- fast, convenient online submission

- thorough peer review by experienced researchers in your field

- rapid publication on acceptance

- support for research data, including large and complex data types

- gold Open Access which fosters wider collaboration and increased citations

- maximum visibility for your research: over 100M website views per year

At BMC, research is always in progress.

Learn more biomedcentral.com/submissions 